Anuario de Estudios Medievales

43/1, enero-junio de 2013, pp. 243-258

ISSN 0066-5061

doi:10.3989/aem.2013.43.1.08

\title{
LES USAGES DU SUCRE \\ DANS LE MANUEL DE PHARMACIE DE COHEN AL-'ATTṬAR, MÉDECIN JUIF DU CAIRE AU XIIIE SIÈCLE ${ }^{1}$
}

\author{
THE USE OF SUGAR \\ IN THE PHARMACY MANUAL OF COHEN AL- 'ATTATR, \\ A JEWISH DOCTOR IN 13TH CENTURY CAIRO
}

MOHAMED OUERFELLI

Aix-Marseille Université

CNRS, LA3M-UMR 7298

\begin{abstract}
Résumé: Face à l'absence d'un guide pharmaceutique à l'usage des apothicaires et des marchands de drogues et de boissons, qui tient compte de l'évolution de la pharmacie et du développement de la matière médicale, Cohen al-'Atțār propose un nouveau manuel dans lequel il rappelle non seulement les écrits de ses prédécesseurs, mais il livre également les points de vue de ses contemporains, et surtout ses propres expériences, qu'il mène dans sa boutique du Caire, pour la confection des médicaments et les moyens de les conserver. Ce manuel pharmaceutique, le plus riche en formules médicamenteuses, se distingue également par l'usage massif du sucre dans l'élaboration des recettes. $\mathrm{Ce}$ produit, disponible en grandes quantités en Égypte et en Syrie pendant la seconde moitié du XIIII siècle, se substitue progressivement au miel et devient l'ingrédient principal dans l'élaboration des médicaments et des boissons. Par cette voie et par un effet de mode, il perce le domaine alimentaire et devient l'apanage des cours princières, qui en font un élément indispensable à leurs réjouissances.
\end{abstract}

Mots-clé: Cohen al- 'Atțâr; pharmacie; sucre; médecine; manuel; recette; médicaments.

\begin{abstract}
In the absence of a pharmaceutical guide for the usage of apothecaries and merchants of drugs and drinks, which took into account the evolution of pharmacy and the development of pharmaceutical products, Cohen al-'Atțār wrote a new manual in which he reminded us not only of the writings of his predecessors, but also the point of view of his contemporaries, notably his proper experiences he carried out in his shop in Cairo to make pharmaceutical products and the means to preserve them. This pharmaceutical manual, the richest in medicine formulas, also stands out on account of the extensive use of sugar in the making of recipes. Given the fact that this product was available in large quantities in Egypt and Syria during the second half of the 13th century, it gradually substituted honey to become the principal ingredient in the making of drugs and drinks. Thus, sugar broke through the food world to become the privilege of the royal class, who turned it into one of the essential elements for their festivities.
\end{abstract}

Keywords: Cohen al-'Atțār; pharmacy; sugar; medicine; manual; recipe; medicaments. \footnotetext{
l'Islam.

${ }^{1}$ Abréviations employées: $\mathrm{BnF}=$ Bibliothèque nationale de France; $E I=$ Encyclopédie de
} 
1. Un nouveau guide à la pointe des connaissances pharmaceutiques.- 2. Les usages du sucre et des produits sucriers.- 3. Conclusion.- 4. Bibliographie citée.

Le sucre, par sa nature, par la diversité de ses usages et par l'étendue de ses propriétés bienfaisantes, est sans contredit la substance la plus précieuse pour l'homme, et celle qui mérite le plus de fixer son attention ${ }^{2}$.

Cette phrase de Dutrône La Couture, médecin français du XVIIIe siècle, résume parfaitement le rôle important du sucre dans la médecine et la pharmacopée durant de nombreux siècles. Elle confirme les dires de Guillaume de Tyr (mort en 1184), qui parle d'un produit si précieux et si nécessaire aux hommes pour toutes sortes d'usages, comme pour leur santé, que les marchands le transportent vers les parties les plus reculées du monde ${ }^{3}$. Ces deux positions séparées par cinq siècles confortent les opinions des médecins médiévaux, qui ont abondamment employé le sucre dans leurs préparations médicamenteuses ${ }^{4}$.

Parmi les œuvres médicales ayant accordé une place particulière au sucre dans l'élaboration des médicaments, se distingue un manuel pharmaceutique écrit par un praticien juif du Caire au début de la période mamelouke. Il s'agit d'Abū al-Munā Dāwūd ibn Abī al-Naṣr ibn al-Haffāọ al-Hārūnī alIsrā'îlī, connu aussi sous le nom de Cohen al- 'Atțār ("le prêtre pharmacien") . Contrairement à l'immense diffusion de son guide pharmaceutique, très peu de choses nous sont parvenues sur la vie et la carrière de ce personnage, qui a vécu certainement au Caire. Si la date de sa naissance ne nous est pas connue, sa mort en revanche est située en 1260, date à laquelle il a terminé la rédaction de son manuel d'officine, qu'il a intitulé Minhāğ al-dukkān wa dustūr al-a 'yān fì a'māl wa tarākìb al-adwiya al-nāfi'a lì al- 'abdān ("Le protocole de l'officine et la convention pour la préparation des médicaments nécessaires aux êtres humains"). Il fait partie d'une série de praticiens juifs qui ont vécu au Caire

${ }^{2}$ J.F. Dutrône La Couture, Précis sur la canne, p. 282.

${ }^{3}$ Guillaume de Tyr, Chronique, p. 589: "verum et cannamellas, unde preciosissima, usibus et saluti mortalium necessaria maxime conficitur zachara, unde per institores ad ultimas orbis partes deportatur".

${ }^{4}$ L. Plouvier, L'introduction du sucre, pp. 199-216.

${ }^{5}$ L. Leclerc, Histoire de la médecine arabe, vol. II, p. 215; M. Meyerhof, Notes sur quelques médecins juifs égyptiens, p. 130; idem, Mediaeval Jewish Physicians, p. 456; V. Mazloum Bey, Deux grands traités intéressant l'Égypte, pp. 47-49; J.-P. Bénézet, Pharmacie et médicament, p. 102; M. Ouerfelli, La consommation du sucre, p. 167. 
au XIIIe siècle, où ils ont exercé leur métier ${ }^{6}$; ils étaient sans doute en relation étroite avec les milieux marchands, les épiciers et les vendeurs de boissons ${ }^{7}$.

Écrit au Caire pour lui-même et pour son fils, le manuel de Cohen al- 'Atṭār est abondamment employé dans les hôpitaux et les officines et connaît une remarquable diffusion dans le monde musulman ${ }^{8}$. Lucien Leclerc l'a qualifié de l'un des meilleurs qui nous soient restés en la matière ${ }^{9}$. Preuve de son importance ${ }^{10}$, il en subsiste aujourd'hui un peu plus d'une quarantaine de manuscrits dispersés dans toutes les bibliothèques du monde arabe et $\mathrm{d}^{\prime}$ Europe $^{11}$, entre autres la Bibliothèque nationale de France, qui conserve cinq manuscrits de ce best-seller ${ }^{12}$. Il a été édité à six reprises dont la première à Boulāq, en 1870 et la dernière en $2001^{13}$, et a également fait l'objet d'une recherche du point de vue de l'histoire sociale, publiée récemment par Leigh Chipman ${ }^{14}$.

Pourquoi Cohen al- 'Atṭār a-t-il envisagé la rédaction d'un nouveau manuel alors qu'il en existait déjà de nombreux pouvant répondre aux besoins des professionnels de la pharmacie? Quelle place accorde-t-il au sucre dans ses préparations et quels en sont les usages?

\footnotetext{
${ }^{6}$ M. Meyerhof, Notes sur quelques médecins juifs égyptiens, p. 130.

${ }^{7}$ S.D. Goitein, A Mediterranean Society, vol. II, pp. 264-267 ; K. Oulad Hammou, Les droguistes dans le monde mamlouk, pp. 96-97.

${ }^{8} E I$, vol. V, p. 249. L'importance de ce guide ne se dément pas, notamment chez les vendeurs de drogues, jusqu' au milieu du XXe siècle; M. Meyerhof, Mediaeval Jewish Physicians, p. 456.

${ }^{9}$ L. Leclerc, Histoire de la médecine arabe, vol. II, p. 215.

${ }^{10}$ M. Levey, Early Arabic Pharmacology, pp. 58-59; S. Hamarneh, The Climax of Medieval Arabic professional pharmacy, pp. 450-461.

${ }^{11}$ L. Chipman, The World of Pharmacy, pp. 182-184, a dressé une liste de 25 manuscrits complets, de 6 incomplets et de 15 fragments (cf. par exemple les fragments publiés par L. Chipman, E. Lev, Syrups from the apothecary's shop, pp. 137-168). Cet inventaire n'est certainement pas exhaustif, dans la mesure où cet auteur ne connaît pas le travail de A.A. al-Difā' Ishām ulamā' al- 'Arab, pp. 416-417, qui signale plusieurs manuscrits dans la Bibliothèque du musée iraquien de Bagdad et à Damas; cf. également $E I$, vol. V, p. 249. Nous avons consulté un manuscrit de la Bibliothèque royale du Maroc, Rabat (cote 314), recueil de cinq traités de médecine et de pharmacopée. Cet ensemble fut copié tardivement par ordre du sultan du Maroc alḤasan I. Le Minhāğ al-dukkān vient en quatrième position et compte 102 feuillets (ff. 51-153).

${ }^{12} \mathrm{BnF}$, Arabe $\mathrm{n}^{\circ} 2965$ (ff. 123v-234r: nous avons consulté cet exemplaire), n² 2993, n 2994, $\mathrm{n}^{\circ} 2995$ et $\mathrm{n}^{\circ} 2996$.

${ }^{13}$ Abū 'l-Munā al-Kūhīn al- 'Atṭār, Minhāğ al-dukkān. Les autres éditions datent de 1883, 1887, 1912 et 1992.

${ }^{14}$ L. Chipman, The World of Pharmacy.
} 


\section{UN NOUVEAU GUIDE À LA POINTE DES CONNAISSANCES PHARMACEUTIQUES}

Les premiers guides pharmaceutiques rédigés par des praticiens tels que Sābūr ibn Sahl ${ }^{15}$ ou al-Kindīin ont montré l'enrichissement de la matière médicale et l'apport de nouveaux produits d'Extrême-Orient. Les grands médecins du Xe siècle, tels que al-Rāzī (Razès) ${ }^{17}$, al-Mağūsī (Haly Abbas $)^{18}$ et Ibn Sīnā (Avicenne) $)^{19}$, ont consacré dans leurs encyclopédies médicales des livres entiers au traitement de la matière médicale et à la préparation des remèdes. Mais ces écrits s'adressent aux praticiens et sont difficiles à assimiler par les professionnels de la pharmacie, qui préfèrent un répertoire plus simplifié et plus pratique pour répondre aux besoins réels des hôpitaux et des officines. Ibn al-Tilmīt (mort en 560/1165) ${ }^{20}$, fort de son expérience en qualité de médecin du calife abbasside, et surtout de sa direction de l'hôpital al-'Aḍudī²1 , écrit un formulaire pharmaceutique (aqrābātīn) pour pallier les insuffisances du guide de Sābūr ibn Sahl, resté en usage dans tout l'Orient musulman pendant plus de deux siècles. En même temps, il rédige un compendium destiné aux

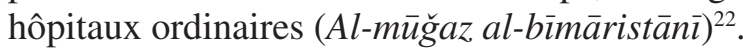

Ce genre d'ouvrage de technique pharmaceutique se diffuse et atteint l'Égypte. Mais il montre rapidement ses limites devant le développement de la fonction de pharmacien hospitalier et la croissance de la matière médicale, ce qui nécessite une mise au point régulière des manuels destinés aux établissements hospitaliers et aux marchands de drogues.

Ainsi au XIIe siècle, Ibn Abī al-Bayān, médecin juif né au Caire en 556/1161 23 , qualifié par Ibn Abī Usaybi ‘a de l'un des plus grands médecins de son siècle ${ }^{24}$, connaît parfaitement la composition des médicaments, leurs doses et leurs propriétés ${ }^{25}$. Son expérience et sa maitrise de la matière médicale l'ont amené à rédiger un guide intitulé Al-dustūr al-bīmāristānī. Il y dé-

${ }^{15}$ Sābūr ibn Sahl, Dispensatorium parvum.

${ }^{16} \mathrm{M}$. Levey, The medical formulary.

${ }^{17}$ Al-Rāzì, Al-Hāwì fi al-tibb, vol. VIII-20: Les médicaments.

${ }^{18}$ Al-Mağūsī, Kāmil al-ṣinā'a al-ṭibbiyya, vol. II-2: la dixième épître sur les médicaments composés.

${ }^{19}$ Ibn Sīnā, Al-Qānūn fì al-țibb, vol. III-5.

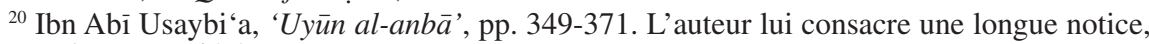
preuve de sa notoriété.

${ }^{21}$ E I, vol. I, p. 355.

${ }^{22}$ Ibn Abī Usaybi'a, 'Uȳ̄n al-anbā', p. 371.

${ }^{23}$ P. Sbath, Le formulaire des hôpitaux d'Ibn Abil Bayan, pp. 13-78; M. Meyerhof, Mediaeval Jewish Physicians, p. 452.

${ }^{24}$ Ibn Abī Usaybi 'a, 'Uyūn al-anbā', p. 584

${ }^{25}$ Ibidem. 
taille toutes les formes de médicaments composés, en usage dans les hôpitaux et dans les officines du monde musulman ${ }^{26}$.

Malgré son succès, ce manuel pharmaceutique est rapidement dépassé; il ne répond plus aux préoccupations des pharmaciens, qui voient la gamme thérapeutique s'élargir et en même temps se compliquer, d'où la volonté de Cohen al-'Atțār de pallier cette insuffisance, en rédigeant un nouveau guide pharmaceutique, qui prenne en compte ses propres expériences et à la fois l'évolution de la médecine et de la pharmacie, et les attentes des professionnels de la santé d'avoir à leur disposition un manuel simplifié et mis à jour.

Tout en reconnaissant de grands mérites au manuel d'Ibn Abì alBayān, Cohen al-'Atṭāar considère qu'aucun guide ne permet de satisfaire les interrogations, notamment de parfumeurs ou de vendeurs de boissons. Car, nous explique-t-il, Ibn Abī al-Bayān s'adressait à une catégorie socioprofessionnelle bien précise, celle des médecins, qui possédaient déjà des connaissances en la matière, alors que les marchands de drogues, les parfumeurs et les vendeurs de boissons en sont dépourvus ${ }^{27}$. Tout en s'appuyant sur les écrits des anciens, Cohen al-'Atṭār a voulu également livrer ses propres expériences et celles de ses contemporains dans la préparation des médicaments $^{28}$. Sur un total de 819 compositions, Al-dustūr al-bìmāristānì d'Ibn Abī al-Bayān est le plus fréquemment cité dans le manuel de Cohen al- 'Aț̣āan avec 82 occurrences. Al-irš̄a d d'Ibn Ğumay' arrive en deuxième position avec 72 citations, loin devant al-Mağūsī et Ibn al-Tilmìt cités respectivement 44 et 34 fois. En revanche, le médecin bagdadien Ibn Ğazla n'est attesté qu'à sept reprises ${ }^{29}$.

\section{LES USAGES DU SUCRE ET DES PRODUITS SUCRIERS}

Ce nouveau manuel, qui actualise les connaissances sur la matière médicale dans la seconde moitié du XIIIe siècle, tient compte également des réalités économiques et de la disponibilité de certains produits sur le marché égyptien. La production du sucre dans la vallée du Nil connaît une croissance spectaculaire, liée à l'intérêt du pouvoir en place pour les revenus fiscaux élevés, rapportés par le sucre et à l'augmentation de la demande de ce produit aussi bien à l'intérieur qu'à l'extérieur de l'Égypte. Avec ce développement,

\footnotetext{
${ }^{26}$ M. Ouerfelli, Le sucre, pp. 513-514.

${ }^{27}$ Cohen al- 'Atțāar, Minhāğ al-dukkān, p. 10.

${ }^{28}$ M. Meyerhof, Notes sur quelques médecins juifs égyptiens, pp. 130-131.

${ }^{29}$ P.B. Fenton, Le Minhāj ad-dukkān, pp. 82-83; L. Chipman, The World of Pharmacy, pp. $25,30,32$ et 38 .
} 
le sucre supplante peu à peu le miel et devient la substance principale dans la préparation des médicaments et des boissons $\mathrm{s}^{30}$.

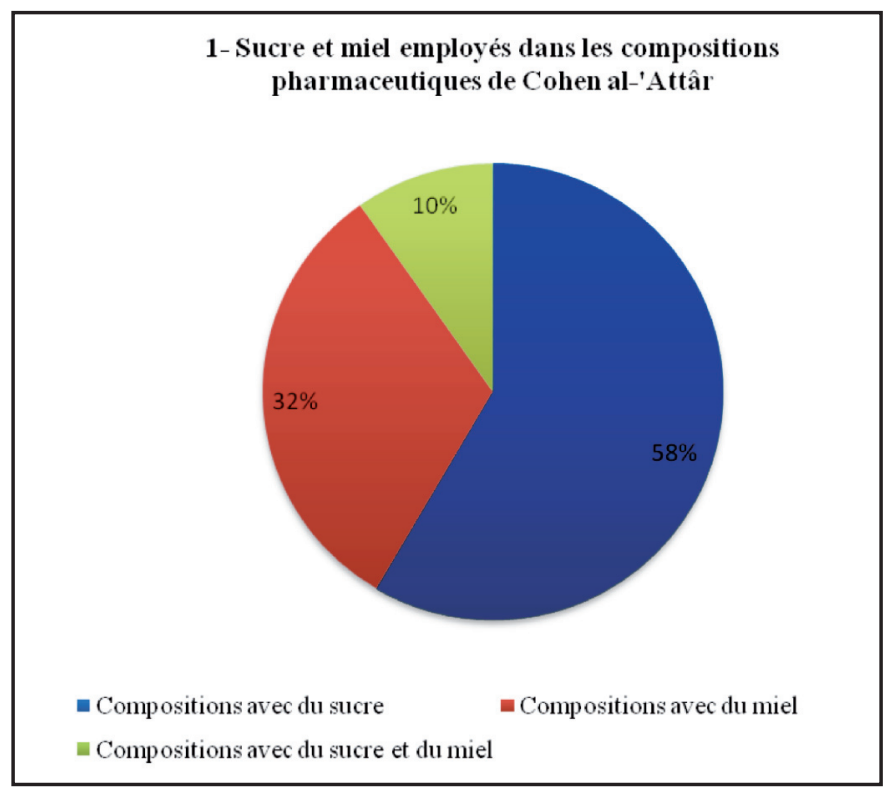

Comme le montre le graphique $\mathrm{n}^{\circ} 1$, le sucre est utilisé dans $58 \%$ des formules contre seulement $32 \%$ où le miel est l'ingrédient principal. Dans 40 compositions, les deux produits sont associés. Le sucre a progressivement réussi à s'imposer, détrônant le miel dans le rôle d'agent conservateur, d'édulcorant et d'excipient. On remarque une forte présence du sucre par rapport au miel notamment dans la composition des sirops. Cohen al-'Atțār explique cette préférence par le fait que le miel possède un pouvoir de conservation assez limité par rapport au sucre, qui permet de garder plus longtemps la consistance des fruits ${ }^{31}$.

Le manuel de Cohen al- 'Atțār met donc en évidence un tournant dans l'emploi du sucre dans le domaine pharmaceutique, mais aussi dans l'alimentation de l'élite sociale égyptienne qui use et abuse de ce produit pour exprimer le luxe et la richesse ${ }^{32}$.

\footnotetext{
${ }^{30}$ M. Ouerfelli, La consommation du sucre, p. 166.

${ }^{31}$ Cohen al-'Aț̣ār, Minhāğ al-dukkān, pp. 300-301.

${ }^{32}$ Sur les usages alimentaires de ce produit, cf. M. Ouerfelli, Le sucre, pp. 598-606.
} 
Avant de passer en revue et d'analyser les différents usages du sucre, il convient d'abord de préciser quelles catégories de sucre emploie Cohen al'Atțār dans son manuel d'officine et en quelle proportion par rapport au miel. Comme il apparaît dans le graphique $n^{\circ} 2$, Cohen al- 'Atțāar préconise l'emploi du sucre blanc dans $64 \%$ des 268 compositions où ce produit est présent. Dans la majorité des cas, il n'indique pas la variété qu'il faut utiliser pour confectionner tel ou tel médicament. Dans seulement 31 formules, il donne plus de précisions et mentionne expressément du sucre blanc à 21 reprises $^{33}$, du sucre pur cinq fois ${ }^{34}$, du sucre en poudre à trois reprises ${ }^{35}$, et enfin, il conseille par deux fois seulement de choisir un produit d'une extrême blancheur ${ }^{36}$. Outre ces quelques indications, le sucre destiné aux usages médico-pharmaceutiques doit être choisi parmi les meilleures qualités produites ${ }^{37}$.

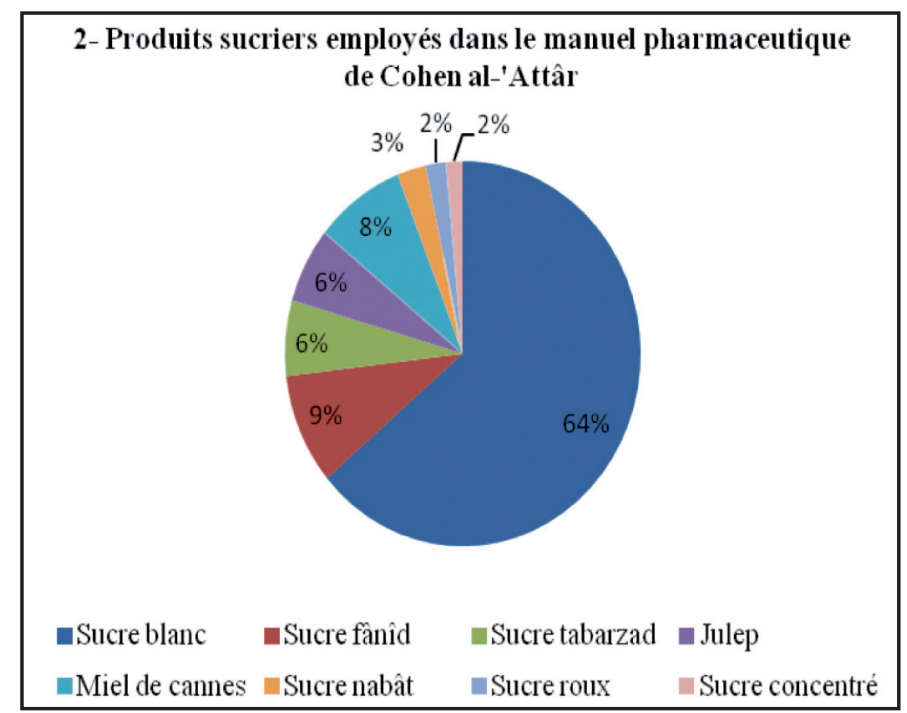

Selon l'anonyme de Kanz al- 'attāar (Le trésor de l'apothicaire), un guide pharmaceutique écrit au Maroc, probablement à la fin du Moyen Âge, parmi les variétés de sucre que l'on utilise dans la médecine et la pharma-

${ }^{33}$ Cohen al-'Aț̣ār, Minhāğ al-dukkān, pp. 35, 41, 44, 45, 49, 52, 53, 58, 61, 62, 75, 79, 87, $89,93,94,95$ et 109 .

${ }^{34}$ Ibidem, pp. 35, 38, 41, 51 et 85.

${ }^{35}$ Ibidem, pp. 41, 61 et 63.

${ }^{36}$ Ibidem, pp. 58 et 62.

${ }^{37}$ M. Ouerfelli, Le sucre, pp. 313-320. 
copée figurent le tabarzad et le fānī ${ }^{38}$. Le premier, employé par Cohen al'Atțār dans 17 formules médicamenteuses, est une bonne qualité de sucre. Le terme tabarzad, d'origine persane passé à l'arabe, comporte deux racines: tabar désigne la hache et zad signifie frappé, c'est-à-dire sucre cristallisé, dur à casser ou à rompre ${ }^{39}$. Pour obtenir cette qualité, soit un sucre d'une extrême blancheur, on cuit le jus de cannes plusieurs fois, en y ajoutant de la crème de lait. Le médecin badgadien Ibn Ğazla (mort en 1100) préconise d'ajouter dix ratls de crème de lait pour cent ratls de sucre ${ }^{40}$. Ainsi, le tabarzad est très recherché par les médecins qui l'emploient souvent dans leurs préparations, comme l'indique Maïmonide: quand les médecins parlent de sucre tabarzad, ils veulent dire sucre solide et dur ${ }^{41}$.

Quant à la seconde catégorie, le fānìd, que Cohen al-'Atțār prescrit dans 25 formules, est un sucre pur de trois cuissons ${ }^{42}$. Le terme est passé lui aussi du persan à l'arabe; le lieu d'origine de cette qualité est le sud-est iranien, la province de Makrān, d'où ce sucre fin et blanc est exporté vers tous les horizons ${ }^{43}$. Appellation et techniques de fabrication viennent ainsi de cette région, avant d'être adoptées en Syrie et en Égypte.

Lorsque le sucre subit une quatrième cuisson avec l'ajout d'ingrédients tels que de l'huile d'amande douce, on l'appelle alors nabāt. Si le tabarzad n'est connu que par les livres de médecine et de pharmacopée, le sucre nabāt en revanche est attesté dans la documentation commerciale. Il figure par exemple parmi les marchandises importées, pendant la seconde moitié du XIIe siècle, dans le royaume de Jérusalem par mer et par voie de terre ${ }^{44}$. Au début du XIVe siècle, Zibaldone da Canal range cette catégorie de sucre parmi les objets du commerce à Alexandrie ${ }^{45}$. Par la suite, le sucre nabāt, très recherché par les marchands occidentaux sur les places de Syrie et d'Égypte, ne disparaît pas mais il prend une autre désignation: celle de sucre candi, terme qui vient

${ }^{38}$ Anonyme, Kanz al- 'attār, Rabat, Bibliothèque royale du Maroc, ms. n 1617, ff. 19-20: l'auteur de ce guide d'apothicaire évoque également d'autres variétés de sucre en usage dans la médecine et la pharmacopée telles que: l'égyptien, le syrien, le sulaymānī et le sūsī. Il considère que la meilleure qualité est de couleur blanche et fond rapidement.

${ }^{39}$ R. Dozy, Supplément aux dictionnaires arabes, vol. II, p. 20.

${ }^{40}$ Ibn Ǧazla, Minhāă al-bayān, BnF, ms. Arabe n ${ }^{\circ} 2948$, f. 116r-v. Le ratl d'Iraq vaut 406,25 grammes. Cohen al-'Attār ne néglige aucun élément en lien avec son métier de pharmacien; il introduit dans son manuel un chapitre sur les poids et les mesures employés en Égypte à son époque, organisé par ordre alphabétique; Minhāğ al-dukkān, pp. 261-64.

${ }^{41}$ Maïmonide, Šarh asmā' al- 'uqqār, p. 141.

${ }^{42}$ Anonyme, Kitāb tahāb al-kusūf, Rabat, Bibliothèque royale du Maroc, ms. n 314, f. 193r.

${ }^{43}$ Idrīsīi, Nuzhat al-muštāq, vol. I, p. 174.

${ }^{44}$ Assises de Jérusalem, II: Assises de la Cour des Bourgeois, p. 176, article 40: sucre nabeth.

${ }^{45}$ Zibaldone da Canal, Manoscritto mercantile del sec. XIV, pp. 57 et 66. 
du sanscrit khanda signifiant à l'origine morceau ${ }^{46}$. Il ne s'agit donc pas d'un sucre de Candie, en Crète, comme certains ont pu le croire ${ }^{47}$, ni de qand, qui est le jus de canne cristallisé, mais plutôt du nabā $t^{48}$. Il se présente sous forme de gros morceaux cristallisés ${ }^{49}$. À en croire Pegolotti, le meilleur et le plus fin sucre candi est celui qui est gros, blanc, clair et sans petits morceaux ${ }^{50}$.

Ces sucres de meilleure qualité, commercialisés en Égypte et exportés vers les marchés de l'Europe, sont appréciés par les apothicaires et les professionnels de la santé pour leurs préparations médicamenteuses. Mais ils ne sont pas toujours fabriqués dans les règles de l'art. Cohen al- 'Atțār recommande à son fils d'employer des produits de bonne qualité et de veiller au bon dosage dans ses compositions ${ }^{51}$. Ibn al-Hāğğ, maghrébin installé au Caire (mort en 1336), met en garde contre les contrefaçons dans l'élaboration des boissons; il remarque qu'à son époque beaucoup de professionnels préparent des sirops et des boissons avec du sucre de médiocre qualité, notamment avec du sucre roux ${ }^{52}$, que Cohen al- 'Atțār n'emploie qu'à cinq reprises ${ }^{53}$. La présence du miel de cannes est également attestée dans le formulaire pharmaceutique à 22 reprises.

Parmi les produits sucriers employés dans le Minhāğ al-dukkān, figure enfin le julep; composé de deux mots, jul (rose) et āb (eau), ce terme d'origine persane est entré dans la langue arabe. Il s'agit d'un médicament liquide, préparé à base de sucre et non de miel $^{54}$. En général, les formules de julep indiquées par les médecins médiévaux devaient ressembler quelque peu aux sirops proprement dits. Mais le julep se distingue par sa légèreté et par sa fluidité, ainsi que par sa consistance variable. Base de toutes les boissons sucrées, Cohen al- 'Atțār l'emploie pour composer de nouvelles formules médicamenteuses, notamment les sirops; il revêt ainsi la fonction d'accompagner des remèdes dont le goût n'est pas agréable, pour atteindre les parties les plus reculées du corps humain. Il le prescrit également pour les traitements de la toux, des fièvres ardentes et des affections pulmonaires ${ }^{55}$. Le julep au citron

\footnotetext{
${ }^{46}$ W. Heyd, Histoire du commerce, vol. II, p. 691, note 11; R. Dozy, Supplément aux dictionnaires arabes, vol. II, p. 641.

${ }^{47}$ Cf. par exemple M. Barceló Crespí, A. Contreras Mas, Farmàcia i alimentació, p. 207.

${ }^{48}$ Al-Nuwayrī, Nihàyat al-'arab, vol. VIII, p. 272.

${ }^{49}$ M. Gual Camarena, El primer manual hispánico, p. 93.

${ }^{50}$ F.B. Pegolotti, La pratica della mercatura, p. 363.

${ }^{51}$ Cohen al-'Aț̣ār, Minhāğ al-dukkān, pp. 269-271.

${ }^{52} \mathrm{Ibn}$ al-Hāğğ, Al-Madhal ilā tanmiyat al-a'māl, vol. IV, pp. 149-150; Kh. Oulad Hammou, Les droguistes dans le monde mamlouk au XVe siècle, pp. 96-97.

${ }^{53}$ Cohen al-'Atțār, Minhāă al-dukkān, pp. 185, 188, 191.

${ }^{54}$ Ibidem, p. 17.

${ }^{55}$ Ibidem, p. 17.
} 
est aussi un excellent désaltérant, qui aide les voyageurs à supporter la chaleur et les longs trajets ${ }^{56}$.

Comme le julep, les sirops entrent dans les préparations liquides qui requièrent d'importantes quantités de sucre. Il s'agit de médicaments liquides, ayant une forte teneur en sucre. Avicenne définit les sirops comme des décoctions ou des sucs épaissis avec une douceur, soit du sucre ou du miel; si le premier n'est pas indispensable, les médecins le recommandent toutefois à la place du miel en raison de sa bonne conservation ${ }^{57}$. Le médecin andalou Abū'l-Qāsim al-Zahrāwī considère que les sirops sont des médicaments agréables et utiles à tous les âges et à tous les moments ${ }^{58}$.

L'élaboration des sirops nécessite une grande quantité de sucre, des eaux distillées et des décoctions. Leur composition varie et peut se faire soit avec une seule matière médicinale, soit avec de nombreux ingrédients. Si le formulaire d'Ibn al-Bayān ne présente que onze recettes de sirops, toutes élaborées avec du sucre ${ }^{59}$, celui de Cohen al- Atțār en revanche est le manuel le plus riche en formules médicamenteuses: il nous livre au total 144 compositions réparties entre sirops, juleps, oxymels et boissons proprement dites, avec une nette prédominance des sirops simples et composés.

3- Produits sucriers employés dans le chapitre des boissons du manuel pharmaceutique de Cohen al-'Attâr

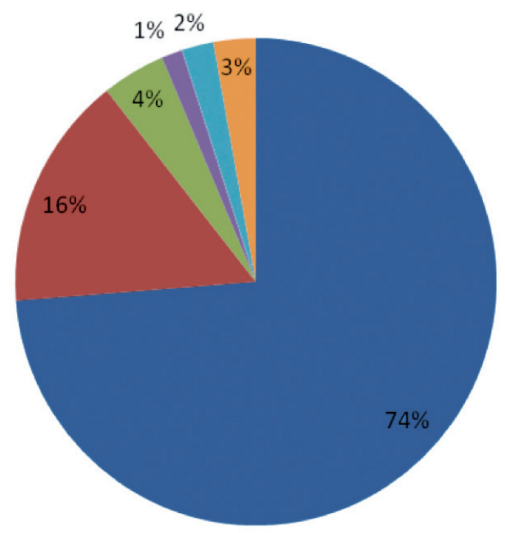

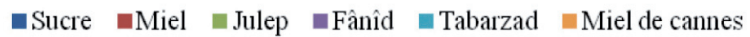

\footnotetext{
${ }^{56}$ Ibidem, p. 19.
}

${ }^{57}$ Ibn Sīnā, Al-Qānūn fì al-tịbb, t. III, p. 363.

${ }^{58}$ Zahrāwī, Extraits, p. 261.

${ }^{59}$ P. Sbath, Le formulaire des hôpitaux, pp. 44-51. 
Dans cette gamme hétérogène, les visées thérapeutiques diffèrent d'une formule à l'autre. Mais les indications les plus fréquentes concernent notamment le traitement des fièvres, les affections des voies respiratoires, les maladies de poitrine et de poumon ${ }^{60}$. Le sirop de grenade par exemple est utile contre les vomissements. Celui de violette est diurétique, efficace contre la toux, la pleurésie, la pneumonie et la néphrite ${ }^{61}$. Le sirop de nénuphar guérit les fièvres aiguës d'origine bilieuse et la toux ${ }^{62}$. Selon Cohen al- Atțāar, le sirop de buglosse est une composition créée par le médecin kairouanais Ibn al-Ğazzār (mort vers 979) pour soigner un jeune homme, souffrant de palpitations, de forte chaleur, de bile jaune à l'estomac et d'insomnie ${ }^{63}$. Les sirops de miel et de sucre ont des propriétés chauffantes: le premier réchauffe l'estomac et le foie, et le second est prescrit contre l'humidité, la pituite; ils conviennent aux personnes de tempérament froid ${ }^{64}$. D'autres sirops sont utilisés contre les vomissements et les diarrhées, tels que le sirop de menthe ${ }^{65}$ ou celui de pomme ${ }^{66}$.

Le skanğabīn ${ }^{67}$, ou sirop de vinaigre, et les sirops acides sont des préparations faites au départ d'eau, de vinaigre et de miel; ensuite les médecins ont employé le sucre sans doute par un effet de mode et par souci d'atténuer l'acidité des médicaments et de les conserver plus longtemps ${ }^{68}$. Ils sont recommandés pour fortifier le foie et l'estomac, pour soigner les fièvres aiguës et pour purifier la bile jaune. À toutes ces indications thérapeutiques, il convient d'ajouter aux sirops des propriétés rafraîchissantes et désaltérantes, d'où leur usage par des personnes qui ne sont pas atteintes de maladies, entre autres par des voyageurs ${ }^{69}$.

Le sucre est aussi employé dans la préparation des robs; ceux-ci désignent des sucs de fruits auxquels il faut ajouter du miel ou du sucre, épaissis par le feu. Cohen al-'Aț̣ār écrit à ce propos:

Le rob est une préparation dans laquelle ne doit pas entrer le sucre en principe, (...) mais en Égypte les fruits sont en général trop

\footnotetext{
${ }^{60}$ Cohen al-'Atțār, Minhāğ al-dukkān, p. 42; d'autres indications thérapeutiques du sucre dans E. Lev, Z. Amar, Practical Materia Medica, pp. 295-296.

${ }^{61}$ Ibidem, p. 32.

${ }^{62}$ Ibidem, p. 17.

${ }^{63}$ Ibidem, p. 43.

${ }^{64}$ Ibidem, p. 51.

${ }^{65}$ Muḥammad al-' Arabi al-Huațāāì Pharmacopée et régimes alimentaires, p. 466.

${ }^{66}$ Anonyme, Aqrabātin al-dukkān, BnF, ms. Arabe n ${ }^{\circ} 3023$, ff. 5v-6r.

${ }^{67}$ Le skanğabìn, terme persan entré dans le vocabulaire médical arabe, se compose de deux mots: sirka, vinaigre et angoubin, miel; c'est l'équivalent de l'oxymel.

${ }^{68}$ Cohen al- 'Atțār, Minhāăg al-dukkān, pp. 20-23.

${ }^{69}$ Ibidem, p. 19.
} 
aqueux et leur suc n'acquiert une consistance suffisante que grâce à l'addition de sucre ${ }^{70}$.

Même s'il conseille par principe de ne pas utiliser ce produit, il reconnaît néanmoins que celui-ci permet une meilleure et plus longue conservation $^{71}$. Il propose d'ailleurs un grand nombre de formules de robs, dont $90 \%$ comportent du sucre ${ }^{72}$.

S'ajoutent à cette catégorie les loochs; ces médicaments préparés sous forme liquide sont composés essentiellement d'une émulsion et d'un mucilage $^{73}$. Leur élaboration se fait avec des racines ou des fruits mucilagineux cuits et mélangés avec du miel, voire du sucre, ainsi qu'avec de l'huile d'amandes douces, ou bien à base de pâte d'amande et de miel. Le sucre est ostensiblement présent parmi les ingrédients: Cohen al- 'Aț̣āar le préconise dans 21 des 32 formules de loochs ${ }^{74}$, et conseille de n'utiliser que les meilleures qualités de sucre, à savoir le fānìd, le nabāt et le tabarzad ${ }^{75}$. Les loochs sont prescrits pour soigner les affections respiratoires, les fièvres, la toux et les lésions des poumons.

On remarque également la présence du sucre avec de fortes proportions dans des préparations de consistance molle, telles que les conserves, les électuaires et les confections. Les premières sont l'équivalent de nos actuelles confitures ${ }^{76}$. Elles servent de matières premières pour la confection d'autres médicaments composés tels les électuaires et les sirops. Les deuxièmes désignent des médicaments à base de substances de choix, et enfin les confections signifient des médicaments achevés. Leurs indications thérapeutiques concernent des troubles digestifs, des douleurs et des fièvres.

Il existe d'autres formes de médicaments composés de consistance ferme dans lesquelles figure le sucre parmi d'autres produits. Sa présence est néanmoins variable d'une forme à l'autre. Il sert non seulement comme excipient, mais aussi pour lier et incorporer les autres drogues. Sur un total de 41 compositions de poudre, Cohen al-'Atțār en propose la moitié avec du sucre

${ }^{70}$ Ibidem, p. 57.

${ }^{71}$ Ibidem, p. 57.

${ }^{72}$ Ibidem, pp. 57-60.

${ }^{73}$ Mucilage: substance végétale composée de pectines, ayant la propriété de gonfler dans l'eau et employée en pharmacie comme excipient médicamenteux et comme laxatif (définition du Petit Robert).

${ }^{74}$ Cohen al-'Aț̣ār, Minhāğ al-dukkān, pp. 105-112.

${ }^{75}$ Ibidem, pp. 105-111.

${ }^{76}$ Ibidem, pp. 61-63: 23 recettes. 
blanc et candi ${ }^{77}$. En revanche, il ne le propose que rarement dans les chapitres des pastilles ${ }^{78}$ et des pilules, uniquement pour donner un goût agréable au médicament.

\section{CONCLUSION}

Force est de constater que la percée spectaculaire du sucre dans la médecine et la pharmacie a donné lieu à un nombre impressionnant de compositions médicamenteuses, dans lesquelles ce produit figure en qualité de principe actif, d'édulcorant, d'excipient, mais aussi d'agent conservateur. Il devient ainsi une composante essentielle, un produit de base pour les pharmaciens et les apothicaires. Son absence ou sa pénurie suscite l'interrogation d'un poète iranien installé à Bagdad au Xe siècle, qui s'est exclamé: À quoi sert une armoire où on ne trouve pas de sucre! $!^{79}$. Une expression équivalente est née en France au début de l'époque moderne, celle d' "apothicaire sans sucre", pour désigner un homme qui manque des objets de première nécessité pour exercer sa profession ${ }^{80}$.

C'est surtout dans les sirops que l'on constate le rôle remarquable du sucre au point de détrôner le miel. Son emploi favorise une meilleure conservation, sujet qui représente un véritable souci pour les médecins et les apothicaires. Son usage donne de surcroît aux sirops un goût agréable; l'ajout de blanc d'œuf lui confère une couleur attrayante. Agréable par le goût et attrayant par la couleur, ce type de médicament a joui et jouit encore de nos jours d'une grande popularité. Par cette voie de la pharmacie et de la médecine, le sucre entre progressivement dans l'alimentation et devient l'apanage des cours princières, qui en font un élément important de leurs banquets et de leurs réjouissances.

${ }^{77}$ Ibidem, pp. 87-95.

${ }^{78}$ Ibidem, pp. 97-104.

${ }^{79}$ Ibrāhīm al-Bayhaqī, Kitāb al-maḥāsin, vol. II, p. 196.

${ }^{80}$ P. Dorveaux, Apothicaires sans sucre, pp. 175-178. 


\section{BIBLIOGRAPHIE CITÉE}

\section{SOURCES}

Abū 'l-Munā al-Kūhīn al-'Ațtā̄r, Minhāğ al-dukkān wa dustūr al-'a yāen, éd. Halīl Ḥasan Ibrāhīm al-Ḥamawī, Beyrouth, 2001.

Anonyme, Aqrabāthīn al-dukkān, BnF, ms. Arabe ${ }^{\circ} 3023$.

Anonyme, Kitāb tahāb al-kusūf, Rabat, Bibliothèque royale du Maroc, ms. $\mathrm{n}^{\circ} 314$.

Assises de Jérusalem, II: Assises de la Cour des Bourgeois, éd. comte Beugnot, Paris, 1843.

Cohen al-'Atțār, Minhāğ al-dukkān wa dustūr al-a yān fì a 'māl wa tarākìb aladwiya al-nāfi'a lì al- 'abdān (Le protocole de l'officine et la convention pour la préparation des médicaments nécessaires aux êtres humains), éd. 'Āsī, Hassan al-, Beyrouth, 1992.

Gual Camarena, Miguel, El primer manual hispanico de mercaderia (siglo XIV), Barcelone, CSIC, 1981.

Guillaume de Tyr, Chronique, éd. R. B. C. Huygens, Turnhout, Brepols, 1986. Ibn Abī Usaybi'a, 'Uyūn al-anbā' fi tabaqāt al-atibbbā', Beyrouth, 1965.

Ibn Ğazla, Minhāă al-bayān fimā yasta'miluhu al-insān (Le Chemin de l'exposition des produits employés par l'homme), BnF, ms. Arabe $\mathrm{n}^{\circ} 2948$.

Ibn al-Hāğğg, Al-Madḥal ilā tanmiyat al-a 'māl bi-tahsīn al-niyāt, le Caire, 1929,4 vols.

Ibn Sīnā, Al-Qānūn fì al-țibb, éd. de Boulaq, s. d., 3 vols.

Ibrāhīm al-Bayhaqī, Kitā̄b al-mahāsin wa-l-masāwi', le Caire, 1906, 2 vols.

Idrīsī, Nuzhat al-muštāq fì 'ihtirā q al-âfā $q$, le Caire, 1994, 2 vols.

Levey, Martin, The medical formulary or aqrābādhin of al-Kindī, Madison, University of Wisconsin Press, 1966.

Maïmonide, Šarh asmā' al- 'uqqār , éd. Max Meyerhof, le Caire, 1940.

Mağūsī, al-, Kāmil al-șinā 'a al-țibbiya, reprod. du ms. AY 4713 de la Bibliothèque universitaire d'Istanbul, éd. F. Sezgin, Francfort, 1985, vol. II-2.

Muhammad al-'Arabi al- Hatṭāaī, Pharmacopée et régimes alimentaires dans les auvres des auteurs hispano-musulmans, Beyrouth, Dar Al-Gharb Al-Islami, 1990.

Nuwayrī, al-, Nihāyat al- 'arab fí funūn al-adab, le Caire, 2007, vol. VIII.

Rāzì, al-, Al-Hâầ fì al-tibb, Beyrouth, 2000, vol. VIII-20.

Pegolotti, Francesco Balducci, La pratica della mercatura, éd. A. Evans, Cambridge (Mass.), 1936. 
Sābūr ibn Sahl, Dispensatorium parvum (al-Aqrābāthīn al-sag̀ìr), éd. O. Kahl, Leyde-NewYork-Cologne, 1994.

Sbath, Paul, Le formulaire des hôpitaux d'Ibn Abil Bayan, médecin du bimaristan an-Nacery au Caire au XIIIe siècle, "Bulletin de l'Institut d'Égypte" 15 (1933), pp. 13-78.

Zahrāwī, Abū'l-Qāsim al-, Extraits du Kitāb al-tasrīf liman 'ağaza 'an alta'lif, dans Pharmacopée et régimes alimentaires dans les cuvres des auteurs hispano-musulmans (en arabe), éd. M. A. al-Hatțābī, Beyrouth, 1990, pp. 237-302.

Zibaldone da Canal, Manoscritto mercantile del sec. XIV, éd. A. Stussi, Venise, Comitato per la pubblicazione delle fonti relative alla storia di Venezia, 1967.

\section{ÉTUDES}

Encyclopédie de l'Islam, nouvelle édition, Leyde, Brill, 2007.

Barceló Crespí, Maria; Contreras Mas, Antoni, Farmàcia i alimentació: L'exemple del sucre a la Mallorca baixmedieval, "Bolletí de la Societat Arqueològica Lul-liana" 50 (1994), pp. 199-218.

Bénézet, Jean-Pierre, Pharmacie et médicament en Méditerranée occidentale (XIIIe-XVIe siècles), Paris, Honoré Champion, 1999.

Chipman, Leigh, The World of Pharmacy and Pharmacists in Mamlûk Cairo, Leyde, Brill, 2011.

Chipman, Leigh; Lev, Efraim, Syrups from the apothecarys shop: a Genizah fragment containing one of the earliest manuscripts of Minhāj aldukkān, "Journal of Semitic Studies" 51/1 (2006), pp. 137-168.

Difā', Abd al-'Azìz al-, Ishām ulamā' al- 'Arab wa-l-Muslimin fì' l-șaydala (L'apport des Arabes et des Musulmans dans la pharmacie), Beyrouth, 1985.

Dorveaux, Paul, Apothicaires sans sucre, "Bulletin des sciences pharmacologiques" 3 (1911), pp. 175-178.

Dozy, Reinhart, Supplément aux dictionnaires arabes, Leyde, Brill, 1967.

Dutrône La Couture, Jacques-François, Précis sur la canne et sur les moyens d'en extraire le sel essentiel. Suivi de plusieurs mémoires sur le sucre, sur le vin de canne, sur l'indigo, sur les habitations et sur l'état actuel de Saint-Domingue, Paris, chez Debure, rue Serpente : chez De Seine, au Palais-Royal, 1791.

Fenton, Paul B., Le Minhāj ad-dukkān d'Abū l-Munā Da'ūd al-Isrā'ìlì. Contribution à l'histoire de la pharmacologie, dans Mélanges d'Histoire de la Médecine hébraïque. Études choisies de la Revue d'Histoire de la Médecine hébrä̈que (1948-1985), Leyde, Brill, 2003, pp. 81-89. 
Goitein, Shelomo Dov, A Mediterranean Society. The Jewish Communities of the World as Portrayed in the Documents of the Cairo Geniza, vol. II: The Community, Berkeley - Los Angeles, University of California Press, 1971.

Hamarneh, Sami, The Climax of Medieval Arabic professional pharmacy, "Bulletin of the History of Medecine" 42 (1968), pp. 450-461.

Heyd, Wilhem, Histoire du commerce du Levant au Moyen Âge, trad. F. Raynaud, Leipzig, 1923, rééd. Amsterdam, Adolf M. Hakkert, 1959, 2 vols.

Leclerc, Lucien, Histoire de la médecine arabe, Paris, E. Leroux, 1876, 2 vols.

Lev, Efraim; Amar, Zohar, Practical Materia Medica of The Medieval Mediterranean According to The Cairo Genizah, Leyde, Brill, 2008.

Levey, Martin, Early Arabic Pharmacology : An introduction Based on Ancient and Medieval Sources, Leyde, Brill, 1973.

Mazloum Bey, Vitalis, Deux grands traités intéressant l'Égypte: la pharmacopée de Cohen el Attar; Les mémoires de Savaresy, et leur place dans l'histoire générale de la pharmacie, "Bulletin de la Société d'histoire de la pharmacie" 46 (1925), pp. 45-50.

Meyerhof, Max, Notes sur quelques médecins juifs égyptiens qui se sont illustrés à l'époque arabe, "Isis"12/1 (1929), pp. 113-131.

Meyerhof, Max, Mediaeval Jewish Physicians in the Near East, from Arabic Sources, "Isis" 28 (1938), pp. 432-460.

Ouerfelli, Mohamed, La consommation du sucre dans le monde méditerranéen médiéval, dans Consommation et consommateurs dans les pays méditerranéens (XVIe-XXe), Actes du colloque de Tunis, 7-9 octobre 2004, "Revue tunisienne des sciences sociales", numéro spécial 129 (2005), pp. 163-184.

Ouerfelli, Mohamed, Le sucre: production, commercialisation et usages dans la Méditerranée médiévale, Leyde, Brill, 2008, (coll. The Medieval Mediterranean; 71).

Oulad Hammou, Khalid, Les droguistes dans le monde mamlouk au XVe siècle : milieu social et normes de travail, dans Collard, F ; Samama, E. éds. (eds.), Pharmacopoles et apothicaires : les «pharmaciens» de l'Antiquité au Grand Siècle. Actes des 4èmes Rencontres d'histoire de la médecine, des pratiques et des représentations médicales dans les sociétés anciennes, Troyes, 20-21 janvier, 2006, Paris, 1'Harmattan, 2006, pp. 89-99.

Plouvier, Liliane, L'introduction du sucre en pharmacie, "Revue d'Histoire de la Pharmacie" 47/322, (1999), pp. 199-216.

Fecha de recepción del artículo : enero 2013

Fecha de aceptación y versión final : mayo 2013 